\title{
High-harmonic Generation from Solid Surface Using an Oscillating Mirror Model and Plasma Mirror System for High Contrast Laser Pulse
}

\author{
I Jong Kim*, II Woo Choi, Karol Adam Janulewicz, and Jongmin Lee \\ Advanced Photonics Research Institute and Center for Femto-Atto Science and Technology, GIST, \\ Gwangju 500-712, Korea
}

(Received January 5, 2009 : revised February 17, 2009 : accepted February 27, 2009)

\begin{abstract}
High-order harmonic generation from a solid surface affected by the contrast of a laser pulse was studied using an oscillating mirror model. High-order harmonics generated from solid surfaces have unusual properties such as spectral redshift, and an intensity difference between even- and odd-order harmonics which is not reported for high-order harmonics generated by a gas medium. We confirmed that high-order harmonics from solid surfaces have selectivity of polarization as well as cut-off extension and the enhancement of conversion efficiency proportional to laser intensity. And the principle of operation and the characteristics of a plasma mirror system developed for achieving high contrast laser pulses to pursue the experimental realization of high-harmonic generation from solid surfaces are reported. Energy fluence on the plasma mirrors is tunable between $10 \mathrm{~J} / \mathrm{cm}^{2}$ and $1000 \mathrm{~J} / \mathrm{cm}^{2}$ and around 1000 shots are available before the plasma mirrors require replacement.
\end{abstract}

Keywords: Plasma mirror, High harmonic generation, Oscillating mirror model, Laser-plasma interaction

OCIS codes : (020.2649) Strong field laser physics; (190.4160) Multiharmonic generation; (320.7080) Ultrafast devices; (340.7480) X-rays

\section{INTRODUCTION}

High field physics has become a topic of extensive research with the development of ultrashort high power lasers. This ultrashort high power laser technology advanced rapidly with the help of chirped-pulse amplification (CPA) [1]. As the laser intensity from the CPA technique is higher than $10^{18} \mathrm{~W} / \mathrm{cm}^{2}$, the relativistic effect should be considered in the laser-plasma interaction. The recent achievement of ultrashort radiation sources generated from the relativistic laser-plasma interaction such as high-harmonic generation (HHG) from solid surfaces [2,3], high energy proton /electron acceleration [4-6] and relativistic nonlinear Thomson scattering (RNTS) $[7,8]$ is significant for many applications. Among them, HHG from solid surfaces is one of the most attractive sources for the generation of $\mathrm{keV}$ radiation in the attosecond time scale. Recently, B. Dromey et al. have a-

*Corresponding author: ijkim@gist.ac.kr chieved harmonic energy of $\sim 17 \mu \mathrm{J}$ at 1200 th $(1.4 \mathrm{keV})$ and $\sim 5 \mu \mathrm{J}$ at 2600 th $(3.1 \mathrm{keV})$ [3]. Y. Nomura et al. have reported that the pulse duration of harmonics generated from a solid surface was 900 attosecond [9].

In this paper, we have calculated HHG from solid surfaces using an oscillating mirror model [10,11] by applying several laser parameters which are available in our laser system. High-order harmonics generated from solids had unusual properties such as spectral redshift and intensity difference between even- and odd-order harmonics, which were not reported for high-order harmonics generated by a gas medium.

However, to achieve high-order harmonics from experiment, it is not enough just to apply ultrashort highintensity laser pulse above $10^{18} \mathrm{~W} / \mathrm{cm}^{2}$. In the ultrashort high power CPA system, the temporal shape of the laser pulse becomes far from Gaussian as the laser energy increases during the amplification. The main pulse is surrounded by a prepulse generated from Amplified Spon- 
taneous Emission (ASE) and pedestal generated by ASE and imperfect compression. The contrast of the pulse is defined by the ratio between the intensity of the main pulse and that of the prepulse/pedestal. When the laser power is low, the prepulse and pedestal play no significant role because they are usually several orders of magnitude less intense than the main pulse, and their intensities are below the damage threshold of the material and the limit of plasma formation. However, when the prepulse and pedestal becomes intense enough, for example, at an intensity of $\sim 10^{12} \mathrm{~W} / \mathrm{cm}^{2}$ for fused silica, they start to damage the material and generate plasma. If the plasma is generated before the main pulse arrival, it will expand hydrodynamically. In other words, the main pulse will no longer interact with plasma having a clean, steep density profile. This results in the limitation of HHG from a solid surface. The degree of contrast is one of the most crucial parameters to decide the characteristic of ultrashort radiation sources generated from the relativistic laser-plasma interaction. Therefore, it is extremely significant to keep the prepulse and pedestal intensity low for the high-intensity laser-plasma interaction.

There are several approaches to improve the contrast of the laser pulse. Among the several approaches such as cross-polarized wave and optical parametric chirped pulse amplification, the usage of a plasma mirror is one of the most excellent methods to achieve high contrast laser pulses. A plasma mirror is used to improve the laser pulse after pulse compression, and the mirror provides higher throughput without limitation on the input laser energy [12-14]. Furthermore, the plasma mirror can be implemented in a laser system without any modification of the system itself, since it is used after the whole CPA chain. In this paper we have reported a plasma mirror system installed in $100 \mathrm{TW}$ Ti:sapphire laser system of Advanced Photonics Research Institute (APRI).

This paper is focused on the principle of operation and the characteristics of a plasma mirror system for achieving high contrast laser pulses to pursue the experimental realization of HHG from solid surfaces calculated by the oscillating mirror model. In part II we report the calculation result on HHG from solid surfaces using an oscillating mirror model. In part III we describe the characteristics of a plasma mirror system installed in APRI to achieve high contrast laser pulses.

\section{HIGH HARMONIC GENERATION FROM SOLID SURFACES USING AN OSCILLATING MIRROR MODEL}

When the femtosecond laser pulse is incident on a solid surface, the laser pulse pushes electrons back into the solid surface. Ions in the solid, on the other hand, do not move on the femtosecond time scale. Thus, the electrons pushed back into the solid by the incident laser pulse are pulled back by the restoring force from the ions. This results in oscillation of the electrons, periodic in the frequency of the incident laser pulses. When the intensity of the incident laser pulse is high enough, the maximum velocity of the electrons approaches the speed of light, and the electron motion becomes relativistic. Since the incident laser pulse is reflected at the electron surface which is moving relativistically, the incident laser pulse experiences the relativistic Doppler effect, thereby generating a frequency component much higher than the original laser pulse.

The process of HHG from plasma surface is based on the interaction between an intense laser pulse and a dense medium such as a solid. An important parameter that characterizes the relativistic interaction is the normalized amplitude $a_{0}$ of the vector potential, which is defined as

$$
a_{0}=\frac{e A}{m_{e} c}
$$

where $e$ is the charge of an electron, $A$ is the vector potential of the laser pulse, $m_{e}$ is the mass of an electron, and $c$ is the speed of light. When the dimensionless parameter $a_{0}$ reaches 1 , the velocity of the electrons becomes comparable to the speed of light. In other words, this parameter defines the relativistic threshold : $a_{0}>1$ means the relativistic interaction, while $a_{0}<1$ means a non-relativistic one. The parameter $a_{0}$ is related to the laser intensity $I$ in a form

$$
I \lambda^{2}=1.37 \times 10^{18} \mathrm{Wcm}^{-2} \mu \mathrm{m}^{2} \times a_{0}^{2}
$$

where $\lambda$ is the wavelength of the laser pulse in $\mu m$ unit and $I$ is the intensity in $\mathrm{W} / \mathrm{cm}^{2}$ unit. This means that intensity of $\sim 2 \times 10^{18} \mathrm{Wcm}^{-2}$ is required to reach the relativistic regime at $800 \mathrm{~nm}$ wavelength.

The scheme for HHG from solid surfaces adapting an

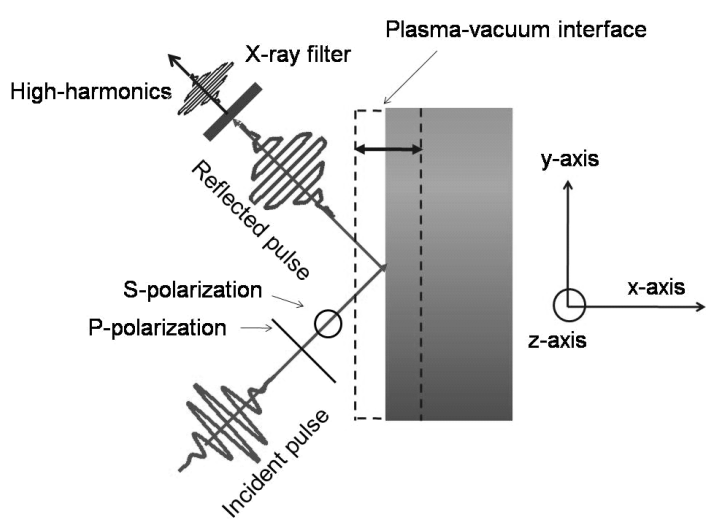

FIG. 1. Scheme for HHG from solid surface driven by an oscillating mirror. 
oscillating mirror is simply denoted in Fig. 1. The laser electric field of the incident pulse drives the plasmavacuum interface back and forth. The laser pulse experiences a large Doppler shift upon the reflection from the oscillating mirror surface, which leads to the generation of the high frequency component. From the relativistic plasma fluid equation, the reflected laser pulse is denoted by the following equations [10]. If the s-polarized laser pulse is incident on a plasma mirror with oblique angle of incidence, the components of reflected field in y-and z-axis $\left(E_{y}(x, t)\right.$ and $\left.E_{z}(x, t)\right)$ during the propagation along the $\mathrm{x}$-axis is as follows,

$$
\begin{aligned}
& E_{y}(x, t)=\frac{\omega_{p}}{2 \omega_{0}}\left[\frac{\sqrt{1-(\dot{\mathbf{X}}(t) / c)^{2}} \times(-\tan \alpha)}{\sqrt{1+\left(\mathbf{a}_{z}\left(\mathbf{X}(t), t_{\text {ret }}\right) \cos \alpha\right)^{2}}}+\tan \alpha\left(1+\frac{\mathbf{X}(t)}{l_{s}}\right)\right] \\
& E_{z}(x, t)=\frac{\omega_{p}}{2 \omega_{0}} \frac{\sqrt{1-(\dot{\mathbf{X}}(t) / c)^{2}} a_{z}\left(\mathbf{X}(t), t_{\text {ret }}\right)}{\sqrt{1+\left(\mathbf{a}_{z}\left(\mathbf{X}(t), t_{\text {ret }}\right) \cos \alpha\right)^{2}}}
\end{aligned}
$$

If the p-polarized laser pulse is incident on the plasma mirror with oblique angle of incidence,

$$
\begin{aligned}
& E_{y}(x, t)=\frac{\omega_{p}}{2 \omega_{0}}\left[\frac{\sqrt{1-(\dot{\mathbf{X}}(t) / c)^{2}}\left(a_{y}\left(\mathbf{X}(t), t_{r e t}\right)-\tan \alpha\right)}{\sqrt{1+\left(\mathbf{a}_{y}\left(\mathbf{X}(t), t_{r e t}\right) \cos \alpha\right)^{2}-a_{y}\left(\mathbf{X}(t), t_{r e t}\right) \sin 2 \alpha}}+\tan \alpha\left(1+\frac{\mathbf{X}(t)}{l_{s}}\right)\right] \\
& E_{z}(x, t)=0
\end{aligned}
$$

where $\mathrm{X}(t)$ is the position vector of $\mathrm{x}$-axis and $\dot{\mathrm{X}}(t)$ is the differentiation of $\mathrm{X}(t)$ with respect to time. $a_{\mathrm{y}}$ and $\mathrm{a}_{\mathrm{z}}$ is the driving field in the y-and z-axis. $a_{y}$ and $a_{z}$ are the components of driving field for the p-polarization and s-polarization respectively. And $\omega_{p}$ is plasma frequency, $\omega_{0}$ is angular frequency of the laser pulse, $\alpha$ is the angle of incidence, and $l_{s}$ is the skin depth. The retarded time $t_{\text {ret }}$ is given by

$$
t_{r e t}=t-X\left(t_{r e t}\right) / c+x / c
$$

And the driving field a is given by

$$
\mathbf{a}\left(X\left(t_{\text {ret }}\right), t_{\text {ret }}\right)=\left(2 \omega_{0} \mathbf{a}_{0} P\left(t_{\text {ret }}\right) / \omega_{p}\right) \sin \left(\omega_{0} t-\mathbf{X}(t) / c\right)
$$

where $p$ is the envelope of the incident field. Y- and $\mathrm{z}$-axis are consistent with the directions of $\mathrm{p}$ - and s-polarization respectively during the field propagation along the x-axis. By the Fourier transform of laser pulse $(E)$, we can achieve high harmonic spectra in the frequency domain.

High-harmonic spectra generated from solids are quite different from high-harmonic spectra generated from atomic gas. In the case of HHG from gas, only odd-order harmonics are generated if the linearly polarized fundamental laser pulse interacts with the gas target. The polarization of high-order harmonics corre- sponds to the polarization of the fundamental laser pulse. On the other hand, HHG from a solid has different characteristics as shown in Fig. 2. For oblique incidence of a p-polarized fundamental laser pulse, p-polarized even- and odd-order harmonics are obtained (Fig. 2(a)). For s-polarized laser pulse we can obtain odd-order harmonics with s-polarization (Fig. 2(b)) and even-order harmonics with p-polarization (Fig. 2(c)). This well- known result can be explained by assuming that the collective motion of the electrons near the plasma-vacuum interface is qualitatively similar to the figure-eight-motion $[15,16]$ of a single electron at the relativistic $(a \geq 1)$ regime. From this figure, the oscillating mirror model predicts well the characteristics of polarization selectivity of the generated harmonics from solid $[10,15]$. If the polarization sensitivity of x-ray spectrometer is high enough to distinguish even- and odd-order harmonics generated from s-polarized laser pulse, we can easily verify the polarization selectivity of $\mathrm{HHG}$ from solid.

Angle of incidence $(\alpha)$ of laser pulses is one of the crucial factors to decide spectral characteristics of harmonics generated from solid surfaces. For $\alpha=0$, there is no difference between s- and p-polarization. Considering that the driving field is an odd function such as equation (6), normal incidence of linearly polarized laser pulses leads to only odd-order harmonics with the polarization of the incident laser pulse. For $\alpha>0$, however, even-order harmonics with p-polarization are generated

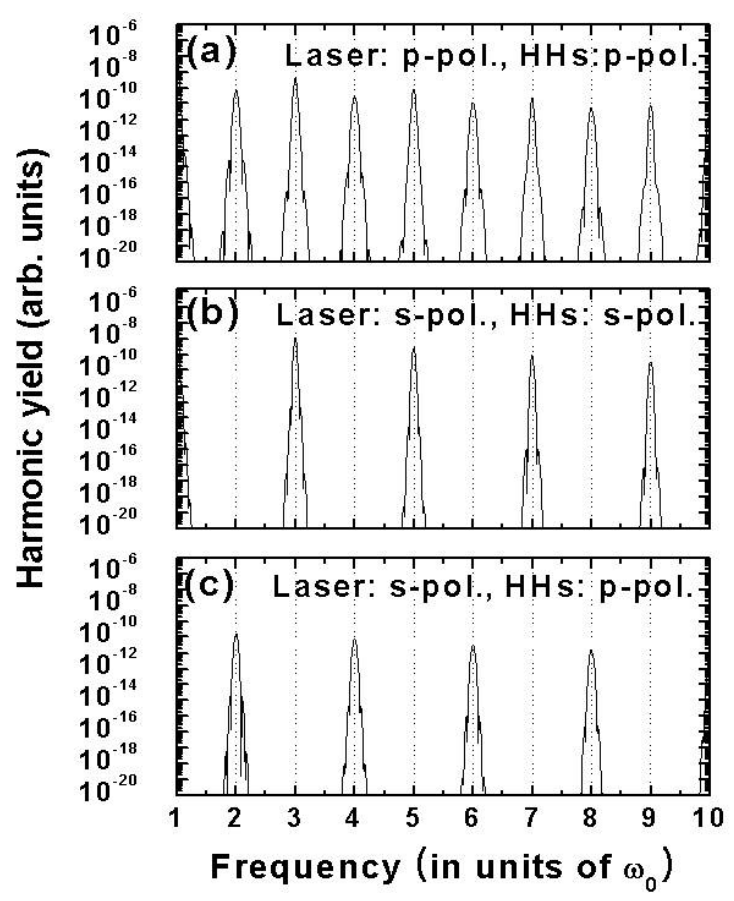

FIG. 2. Calculated harmonic spectra for different polarizations using a laser pulse with $30 \mathrm{fs}, \mathrm{a}_{0}=1$ and $45^{\circ}$ angle of incidence. (a) laser is p-polarized, high-harmonics (HHs) is p-polarized (b) laser is s-polarized, HHs is s-polarized (c) laser is s-polarized, HHs is p-polarized. 


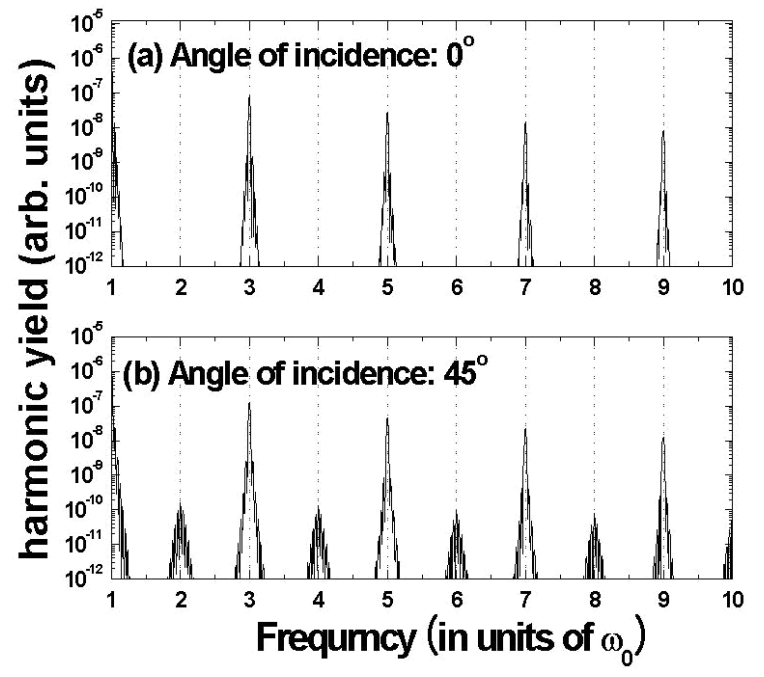

FIG. 3. Calculated harmonic spectra for different angle of incidence with $30 \mathrm{fs}, \mathrm{a}_{0}=10$ and p-polarized laser pulse. (a) is $0^{\circ}$ and (b) is $45^{\circ}$.

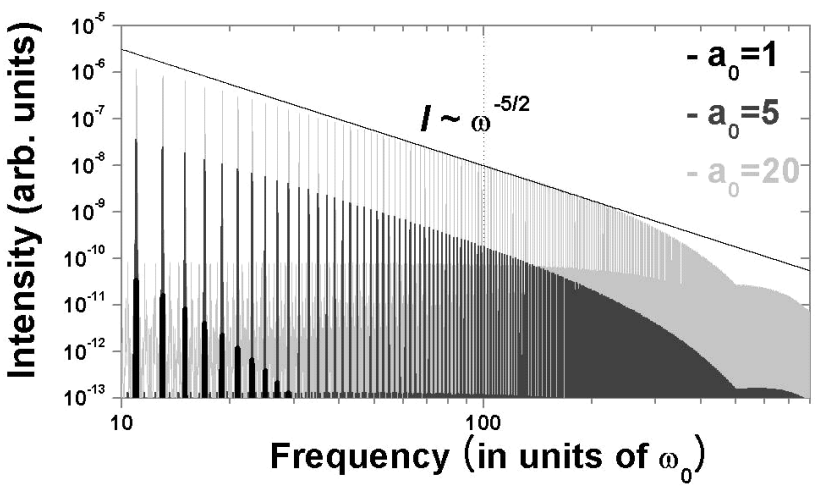

FIG. 4. Calculated harmonic spectra of the reflected radiation for the laser intensity of $\mathrm{a}_{0}=1,5$, and 20 with $30 \mathrm{fs}$, $45^{\circ}$ angle of incidence and s-polarized laser pulse.

irrespective of the polarization of the incident laser pulse. At oblique incidence of $\alpha$, even-order harmonics are weaker than the odd-order harmonics and disappear completely for normal incidence. Moreover, the difference of harmonic yield between even- and odd-orders is larger as the laser intensity is stronger. Figure 3 confirms these results as discussed above.

In general high-order harmonics generated from gas occur in an intensity range of $10^{14} \sim 10^{16} \mathrm{~W} / \mathrm{cm}^{2}$ which is similar to or slightly higher than the magnitude of Coulomb potential of the atom. If the high intensity laser pulse exceeding $10^{17} \mathrm{~W} / \mathrm{cm}^{2}$ is applied to the gas, due to the ionization of the electron before the arrival of the main pulse, the recombination probability of electron to the parent ion does not increase more and even decreases. That is, the effect of ionization plays an important role in limiting the extension of cut-off wavelength and the enhancement of conversion efficiency of high-order harmonics generated from gas. On the other

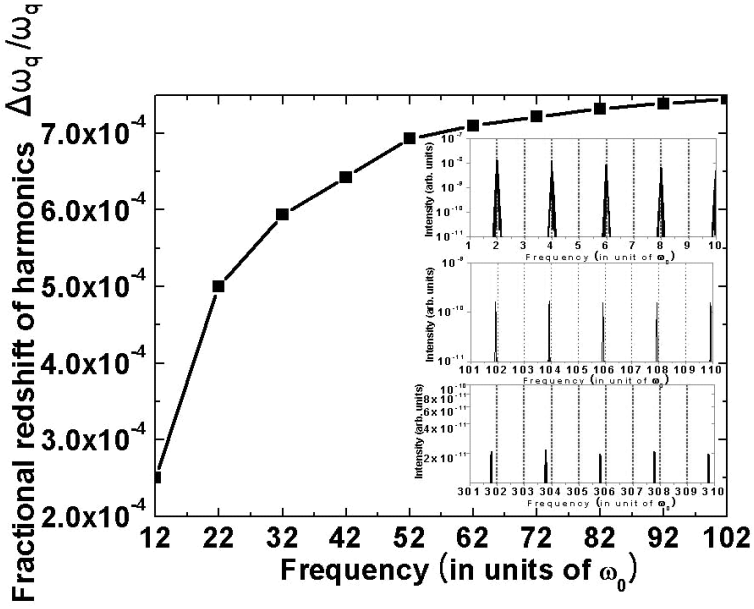

FIG. 5. Spectral redshift of high-order harmonics in a 30 fs, $a_{0}=20$ and s-polarized laser pulse.

hand, HHG from a solid has quite different properties as shown in Fig. 4. There is no limitation to achieving stronger and higher-order harmonics as the laser intensity increases. The stronger the laser intensity, the more the cut-off wavelength is extended and conversion efficiency is enhanced. In addition, the harmonic spectrum follows the scaling law of $\omega_{0}^{-5 / 2}[17]$. If we consider the incident energy of $2 \mathrm{~J}$ and the pulse duration of $35 \mathrm{fs}$, the focused peak intensity is more than $10^{20} \mathrm{~W} / \mathrm{cm}^{2}$. From this, we expect to achieve the conversion efficiency of $10^{-6}$, this corresponds to the energy of $\sim \mathrm{uJ}$ level around $1000^{\text {th }}$.

In HHG from gas, blueshift of high-order harmonics is typical [18]. If we use a laser pulse with a few tens of femtoseconds, due to the rapid increase in the laser electric field as well as the effect by the self-phase modulation in an ionizing gas medium, blueshift is dominant in HHG from gases. On the other hand, spectral redshift $\left(\Delta \omega / \omega<10^{-3}\right)$ of high-order harmonics is observed for HHG from solids (see Fig. 5) with respect to the exact multiples of $\omega_{0}$. Moreover, redshift of harmonic spectra becomes severe as the harmonic order goes higher even if the rate of increment gradually shrinks. This phenomenon is quite new even if the physical origin needs further study.

\section{DOUBLE PLASMA MIRROR SYSTEM IN APRI}

Laser pulse is focused onto a dielectric material. If the intensity of prepulse and pedestal is low enough, they are transmitted without the plasma formation on a target surface. On the other hand, when the main pulse exceeding the damage threshold of the material arrives on the material, plasma is generated on the ionized material surface. As soon as the electron density 
in the plasma exceeds the critical density for the wavelength of the incident laser pulse, the main pulse and everything afterward are reflected. The reflectivity is increased by several orders of magnitude for the main pulse, while it is maintained low for the prepulse and the pedestal. As a result, the contrast of the laser pulse is increased by the ratio of the reflectivity of plasma to the reflectivity of material. Typically, the improvement of contrast is around 2 orders of magnitude for one dielectric material with antireflection (AR) coating.

A double plasma mirror system using two plasma mirrors is established between pulse compressor and target chamber in 100 TW Ti:sapphire CPA system of APRI. The laser pulse with 35-fs pulse duration, 2-J energy and $70-\mathrm{mm}$ beam diameter achieved after the vacuum pulse compressor is incident on the plasma mirror system. After the operation of the plasma mirror, a temporally improved laser pulse with high contrast goes into the target chamber for the laser-plasma interaction. The overall dimension of vacuum chamber for a plasma mirror system is 1600-mm length, 1260-mm width and $680-\mathrm{mm}$ height.

The laser beam after the pulse compressor is incident on a reflective mirror $(120 \times 80 \times 25 \mathrm{~mm})$ with $45^{\circ}$ incidence angle in a plasma mirror system as shown in Fig. 6. The polarization of the laser beam should be changed by periscope A from p-polarization to s-polarization. Also the height of the laser beam is changed from $236 \mathrm{~mm}$ to $366 \mathrm{~mm}$. After the reflection from an off-axis parabolic mirror (OAP) A, the laser beam is focused on the first plasma mirror. OAP has an effective focal length of 1200 $\mathrm{mm}$, and off-axis angle of $20^{\circ}$. Before incidence on the first plasma mirror, we place the folding mirror A between OAP and the plasma mirror to make the vacuum chamber compact. The laser focus formed by the OAP $\mathrm{A}$ is located in the middle of two plasma mirrors, and the angle between the two plasma mirrors is $93.3^{\circ}$. The reason why the angle is not $90^{\circ}$ is that the laser beam is incident on the plasma mirror with $3.3^{\circ}$ with respect to the $\mathrm{x}$-axis. This is because an optical mount manipulating a folding mirror has a certain dimension. Therefore the mount restricts reduction of the interval between two folding mirrors when the edges of two plasma mirrors approach each other closely to get the highest fluence. After the reflection from the second plasma mirror, the laser beam goes to folding mirror B, OAP $\mathrm{B}$, and periscope B. By this process, the laser beam is collimated again and then the beam size is recovered. The laser beam is also p-polarized by the periscope B. The plasma mirror system has symmetry with respect to the $\mathrm{x}$-axis which passes through the laser focus between the two plasma mirrors.

In general, the reflectivity and contrast ratio of the laser pulse reflected from the plasma mirror is quite different according to the polarization of laser pulses incident on the plasma mirror. If the laser pulse with

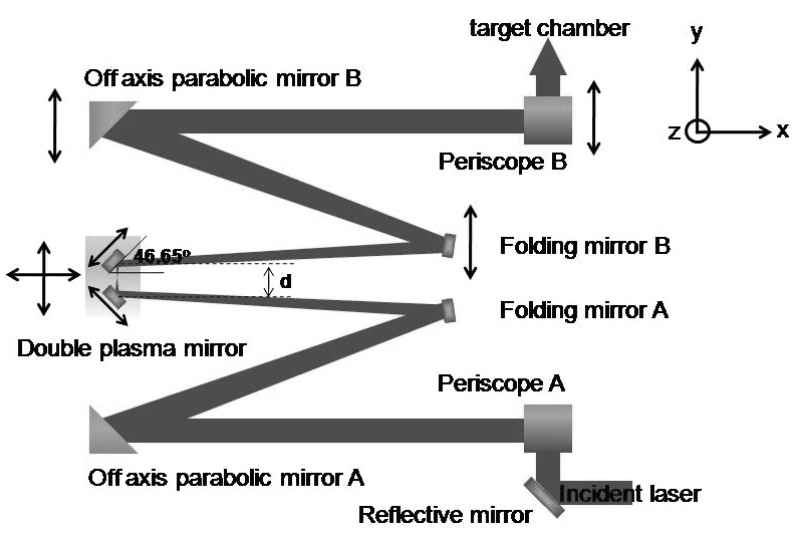

FIG. 6. Schematic diagram of double plasma mirror system. Linear translations of all optical mounts for controlling the energy fluence on plasma mirror are denoted by the arrows.

p-polarization is incident on the plasma mirror, it is well known that the reflectivity and contrast ratio of the laser pulse are decreased remarkably, compared with s-polarized laser pulse, due to the resonance absorption [19]. On the other hand, for the efficient generation of secondary sources such as protons, x-rays and electrons driven by the laser-plasma interaction, the laser pulse with p-polarization is widely used. Because the electron motion which oscillates along the vertical direction with respect to the target surface can be induced when the incident laser pulse has p-polarization. Due to this requirement, we used two periscopes to control the laser polarization. Using these periscopes, the laser polarization is varied from p-polarization to s-polarization, and then comes back to p-polarization again.

The continuous control of energy fluence $\left(\mathrm{J} / \mathrm{cm}^{2}\right)$ incident on the surface of the plasma mirror is one of the most crucial operations in the plasma mirror system. When we assume that the laser beam with the energy of $2 \mathrm{~J}$ and the beam size of $70 \mathrm{~mm}$ is incident in the plasma mirror system, the energy fluence $(E)$ on the plasma mirror is given by the energy per unit area

$$
E=2 /\left(\pi \times r^{2}\right)\left(\text { unit is } \mathrm{J} / \mathrm{cm}^{2}\right)
$$

where the incident energy is fixed to $2 \mathrm{~J}$ and beam radius $r$ on the plasma mirror is proportional to $1 / \sqrt{E}$ as shown in Fig. 7 (a). For the energy fluence of $50 \mathrm{~J} / \mathrm{cm}^{2}$ and $200 \mathrm{~J} / \mathrm{cm}^{2}$, the beam radius is 1.13 $\mathrm{mm}$ and $0.56 \mathrm{~mm}$ respectively. From equation 7 , the beam diameter $D=2 r=2 \times 10 \sqrt{2 /(\pi \times E)}$ (unit is $\mathrm{mm}$ ) and the projection $(y)$ to the plasma mirror surface with the angle of $46.65^{\circ}$ with respect to the $\mathrm{x}$-axis such as in Fig. 6 becomes $y=20 \sqrt{2 /(\pi \times E)} \times \cos \left(46.45^{\circ}\right)$. From the proportional relation between the focal length of 1200 $\mathrm{mm}$ and the full beam diameter of $70 \mathrm{~mm}$, we can obtain the distance $d$ approximately between points on the plasma mirrors hit by the laser beam satisfy 


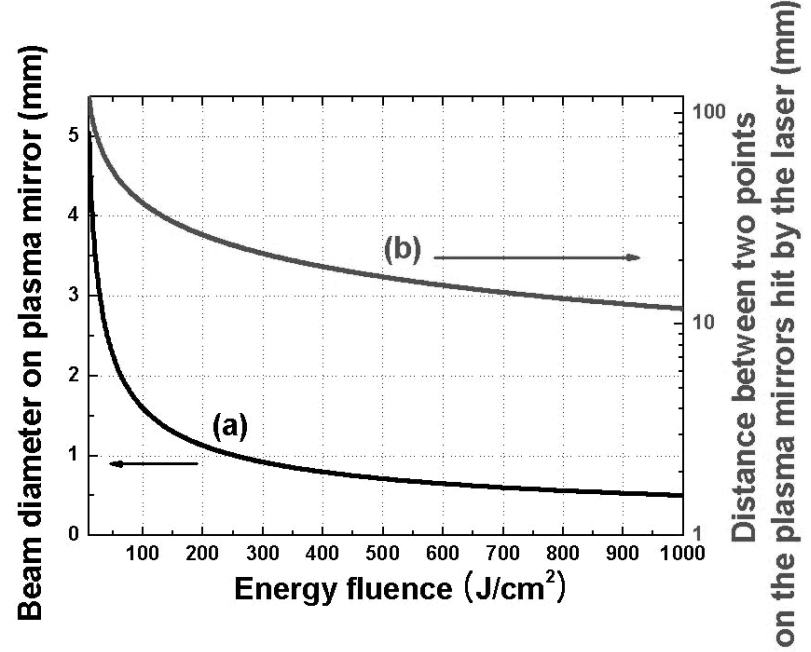

FIG. 7. (a) Beam diameter on plasma mirror and (b) distance between two points on the plasma mirrors hit by the laser beam as a function of energy fluence.

$$
d=2 \times 1200 / 70 \times y=40 \times 1200 / 70 \times \sqrt{2 /(\pi \times E)} \times \cos \left(46.65^{\circ}\right)
$$

(unit is $\mathrm{mm}$ )

$d$ is also proportional to $1 / \sqrt{E}$ as shown in Fig. 7 (b). For example, in the condition of energy fluence of 50 $\mathrm{J} / \mathrm{cm}^{2}$ and $200 \mathrm{~J} / \mathrm{cm}^{2}$, the distance $d$ is $53.11 \mathrm{~mm}$ and $26.56 \mathrm{~mm}$ respectively. Linear translations of all optical mounts for controlling the energy fluence on a plasma mirror are denoted by the arrows in Fig. 6. By moving the necessary optical components, energy fluence can be controlled in a range of $10 \mathrm{~J} / \mathrm{cm}^{2}$ and $1000 \mathrm{~J} / \mathrm{cm}^{2}$.

To enhance the effect of the plasma mirror so that prepulse and pedestal are transmitted and main pulse is reflected, the coating selection on the plasma mirror surface is significant. In addition, for the generation of plasma near the main pulse with high energy fluence, the damage threshold of the plasma mirror should be higher. For this requirement, we considered following conditions. Plasma mirror with a size of $30-\mathrm{mm}$ width, 200-mm length and 25-mm thickness is made of fused silica. We use an antireflection (AR) coating on fused silica. To facilitate the alignment of the whole plasma mirror system, high reflection coating with $10-\mathrm{mm}$ width is deposited on the center of the plasma mirror.

Another issue is that the center wavelength of the ASE pedestal is $786 \mathrm{~nm}$ whereas that of the main pulse is $800 \mathrm{~nm}$. Since the ASE pedestal arrives earlier than the main pulse on the plasma mirror, if the coating of the plasma mirror is matched well with the center wavelength of the ASE pedestal and its reflectivity for the wavelength range of the main pulse between 740 $\mathrm{nm}$ and $840 \mathrm{~nm}$ is minimized, the pedestal can be transmitted into the plasma mirror and the main pulse can be reflected more effectively after the plasma formation on the surface of the plasma mirror. Therefore, we applied AR coating with the reflectivity of $0.2 \%$ for the center wavelength of $786 \mathrm{~nm}$ and damage threshold of $15 \mathrm{~J} / \mathrm{cm}^{2}$ on the plasma mirrors.

After each shot, the double plasma mirror is automatically shifted to offer a fresh surface for the next shot. If we search for the condition of the energy fluence so as to have the highest reflectivity by controlling the $\mathrm{x}$ and y-stages of the plasma mirror, the distance (d) between two points on the plasma mirrors hit by the laser beam can be fixed. We can offer a fresh surface for the next shot by controlling the two actuators which move along the surface directions of two plasma mirrors, as shown in Fig. 6.

\section{CONCLUSION}

High-harmonics from solid surfaces have unusual characteristics such as generation of all-order harmonics even with a single color laser, intensity difference between even- and odd-order harmonics, and spectral redshift. And the principle of operation and the characteristics of the plasma mirror system to achieve high contrast laser pulses were reported for pursuing the experimental realization of HHG from a solid surface. A double plasma mirror system which has been designed at APRI is tunable between $10 \mathrm{~J} / \mathrm{cm}^{2}$ and $1000 \mathrm{~J} / \mathrm{cm}^{2}$, and a total of $\sim 1000$ laser shots are available. Now we are planning to pursue a harmonic generation experiment from solid using high-contrast laser pulses driven by a plasma mirror system.

\section{ACKNOWLEDGMENT}

This work was supported by the Ministry of Knowledge and Economy of Korea through the Ultrashort Quantum Beam Facility Program.

\section{REFERENCES}

1. D. Strickland and G. Mourou, "Compression of amplified chirped optical pulses,” Opt. Comm. 56, 219 (1985).

2. B. Dromey, M. Zepf, A. Gopal, K. Lancaster, M. S. Wei, K. Krushelnick, M. Tatarakis, N. Vakakis, S. Moustaizis, R. Kodama, M. Tampo, C. Stoeckl, R. Clarke, H. Habara, D. Neely, S. Karsch, and P. Norreys, "High harmonic generation in the relativistic limit," Nat. Phys. 2, 456 (2006).

3. B. Dromey, S. Kar, C. Bellei, D. C. Carroll, R. J. Clarke, J. S. Green, S. Kneip, K. Markey, S. R. Nage, P. T. Simpson, L. Willingale, P. McKenna, D. Neely, Z. Najmudin, K. Krushelnick, P. A. Norreys, and M. Zepf, "Bright multi-keV harmonic generation form relativistically oscillating plasma surfaces," Phy. Rev. Lett. 99, 085001 (2007). 
4. M. Kaluza, J. Schreiber, M. I. K. Santala, G. D. Tsakiris, K. Eidmann, J. Meyer-ter-Vehn, and K. J. Witte, "Influence of the laser prepulse on proton acceleration in thin-foil experiments," Phys. Rev. Lett. 93, 045003 (2004).

5. M. Nishiuchi, H. Daido, A. Yogo, S. Orimo, K. Ogura, J. Ma, A. Sagisaka, M. Mori, A. S. Pirozhkov, H. Kiriyama, S. V. Bulanov, T. Zh. Esirkepov, I. W. Choi, C. M. Kim, T. M. Jeong, T. J. Yu, J. H. Sung, S. K. Lee, N. Hafz, K. H. Pae, Y.-C. Noh, D.-K. Ko, J. Lee, Y. Oishi, K. Nemoto, H. Nagatomo, K. Nagai, and H. Azuma, "Efficient production of a collimated MeV proton beam from a ployimide target driven by an intense femtosecond laser pulse," Phys. Plasmas 15, 053104 (2008).

6. N. A. M. Hafz, T. M. Jeong, I. W. Choi, S. K. Lee, K. H. Pae, V. V. Kulagin, J. H. Sung, T. J. Yu, K.-H. Hong, T. Hosokai, J. R. Cary, D.-K. Ko, and J. Lee, "Stable generation of $\mathrm{GeV}$-class electron beams from self-guided laser-plasma shannels,” Nat. Photonics 2, 571 (2008).

7. K. Lee, Y. H. Cha, M. S. Shin, B. H. Kim, and D. Kim, "Relativistic nonlinear thomson scattering as attosecond x-ray source," Phys. Rev. E 67, 026502 (2003).

8. K. Lee, B. H. Lee, and D. Kim, "Coherent radiation of relativistic nonlinear thomson scattering," Phys. Plasmas 12, 043107 (2005).

9. Y. Nomura, R. Hörlein, P. Tzallas, B. Dromey, S. Rykovanov, Zs. Major, J. Osterhoff, S. Karsch, L. Veisz, M. Zepf, D. Charalambidis, F. Krausz, and G. D. Tsakiris, "Attosecond phase locking of harmonics emitted from laser-produced plasmas," Nat. Phys. 5, 124 (2009).

10. R. Lichters, J. Meyer-ter-Vehn, and A. Pukhov, "Shortpulse laser harmonics from oscillating plasma surfaces driven at relativistic intensity," Phys. Plasmas 3, 3425 (1996).

11. G. D Tsakiris, K. Eidmann, J. Meyer-ter-Vehn, and F. Krausz, "Route to intense single attosecond pulses," New. J. Phys. 8, 19 (2006).

12. B. Dromey, S. Kar, M. Zepf, and P. Foster, "The plasma mirror-A subpicosecond optical switch for ultrahigh power lasers," Rev. Sci. Instrum. 75, 645 (2004).

13. A. Levy, T. Ceccotti, P. D’Oliveira, F. Reau, M. Perdrix, F. Quere, P. Monot, M. Bougeard, H. Lagadec, and P. Martin, "Double plasma mirror for ultrahigh temporal contrast ultraintense laser pulses," Opt. Lett. 32, 310 (2007).

14. C. Thaury, F. Quere, J.-P. Geindre, A. Levy, T. Ceccotti, P. Monot, M. Bougeard, F. Reau, P. D'oliveira, P. Audebert, R. Marjoribanks, and Ph. Martin, "Plasma mirrors for ultrahigh-intensity optics,” Nat. Phys. 3, 424 (2007).

15. D. von der Linde and K. Rzàzewski, "High-order optical harmonic generation from solid surfaces," Appl. Phys. B 63, 499 (1996).

16. L. D. Landau and E. M. Lifshitz, The Classical Theory of Fields, vol. 2 of Landau and Lifshitz Course of Theoretical Physics (Pergamon Press, Oxford, 1975).

17. S. Gordienko, A. Pukhov, O. Shorokhov, and T. Beava, "Relativistic doppler effect: universal spectra and zeptosecond pulses," Phy. Rev. Lett. 93, 115002 (2004).

18. H. J. Shin, D. G. Lee, Y. H. Cha, K. H. Hong, and C. H. Nam, "Generation of nanadiabatic blueshift of high harmonics in an intense femtosecond laser field," Phys. Rev. Lett. 83, 2544 (1999).

19. J. P. Freidberg, R. W. Mitchell, R. L. Morse, and L. I. Rudsinski, "Resonant absorption of laser light by plasma targets," Phys. Rev. Lett. 28, 795 (1972). 